\title{
Targeted and global pharmacometabolomics in everolimus- based immunosuppression: association of co-medication and lysophosphatidylcholines with dose requirement
}

\author{
Dorothea Lesche $^{1,2} \cdot$ Vilborg Sigurdardottir $^{3}$ - Alexander B. Leichtle ${ }^{1} \cdot$ Christos T. Nakas $^{1,4}$. Uwe Christians ${ }^{5}$. \\ Lars Englberger $^{6} \cdot$ Martin Fiedler $^{1} \cdot$ Carlo R. Largiadèr $^{1} \cdot$ Paul Mohacsi $^{6} \cdot$ Johanna Sistonen $^{1}$
}

Received: 25 September 2017 / Accepted: 4 November 2017 / Published online: 25 November 2017

(c) Springer Science+Business Media, LLC, part of Springer Nature 2017

\begin{abstract}
Introduction The immunosuppressive therapy with everolimus (ERL) after heart transplantation is characterized by a narrow therapeutic window and a substantial variability in dose requirement. Factors explaining this variability are largely unknown. Objectives Our aim was to evaluate factors affecting ERL metabolism and to identify novel metabolites associated with the individual ERL dose requirement to elucidate mechanisms underlying ERL dose response variability.

Method We used liquid chromatography coupled with mass spectrometry for quantification of ERL metabolites in 41 heart transplant patients and evaluated the effect of clinical and genetic factors on ERL pharmacokinetics. Non-targeted plasma metabolic profiling by ultra-performance liquid chromatography and high resolution quadrupole-time-of-flight mass spectrometry was used to identify novel metabolites associated with ERL dose requirement.

Results The determination of ERL metabolites revealed differences in metabolite patterns that were independent from clinical or genetic factors. Whereas higher ERL dose requirement was associated with co-administration of sodium-mycophenolic acid and the CYP3A5 expressor genotype, lower dose was required for patients receiving vitamin $\mathrm{K}$ antagonists. Global metabolic profiling revealed several novel metabolites associated with ERL dose requirement. One of them was identified as lysophosphatidylcholine (lysoPC) (16:0/0:0). Subsequent targeted analysis revealed that high levels of several lysoPCs were significantly associated with higher ERL dose requirement.

Conclusion For the first time, this study describes distinct ERL metabolite patterns in heart transplant patients and detected potentially new drug-drug interactions. The global metabolic profiling facilitated the discovery of novel metabolites associated with ERL dose requirement that might represent new clinically valuable biomarkers to guide ERL therapy.
\end{abstract}

Keywords Pharmacometabolomics $\cdot$ Everolimus metabolism $\cdot$ Heart transplantation $\cdot$ Dose requirement

Dorothea Lesche and Vilborg Sigurdardottir are co-primary authors.

Electronic supplementary material The online version of this article (https://doi.org/10.1007/s11306-017-1294-8) contains supplementary material, which is available to authorized users.

Paul Mohacsi

paul.mohacsi@insel.ch

1 University Institute of Clinical Chemistry, Inselspital, Bern University Hospital, University of Bern, Bern, Switzerland

2 Graduate School for Cellular and Biomedical Sciences, University of Bern, Bern, Switzerland

3 Department of Cardiology, Swiss Cardiovascular Centre, Inselspital, Bern University Hospital, University of Bern, Bern, Switzerland
4 Laboratory of Biometry, University of Thessaly, Volos, Greece

5 iC42 Clinical Research and Development, University of Colorado, Anschutz Medical Campus, Aurora, CO, USA

6 Department of Cardiovascular Surgery, Swiss Cardiovascular Centre, Inselspital, Bern University Hospital, University of Bern, Bern, Switzerland 


\section{Introduction}

Immunosuppressive therapy plays an essential role in the prevention of allograft rejection after heart transplantation (Azimzadeh et al. 2011). Since the use of calcineurin inhibitors is limited by specific adverse effects like nephrotoxicity, newer drugs such as the mammalian target of rapamycin inhibitor everolimus (ERL) have been introduced to the immunosuppressive therapy (Eisen et al. 2003). Due to its renal-sparing mode of action and anti-proliferative effects slowing the progression of cardiac allograft vasculopathy (CAV; the major long-term complication in cardiac transplant recipients), ERL has become an alternative immunosuppressant for these patients over recent years (Andreassen et al. 2014; Raichlin and Kushwaha 2008). Nevertheless, administration of ERL is complicated by unpredictable inter- and intraindividual variability in dose requirement and therapy response (Kovarik et al. 2003). To gain insight into the variability in ERL dose response, pharmacogenetic studies of genes encoding for proteins involved in the metabolism and transport of ERL have been conducted (Lemaitre et al. 2012; Moes et al. 2014; Schoeppler et al. 2014). Similarly to calcineurin inhibitors, ERL is metabolized by enzymes of the cytochrome P450 (CYP) 3A family, CYP3A4 and CYP3A5, and to a lower extent by CYP2C8, resulting in mainly hydroxylated and demethylated metabolites (Fig. 1) (Kirchner et al. 2004). Although the loss-of-function variant $C Y P 3 A 5^{*} 3$ was reported to be associated with reduced ERL dose requirement (Lesche et al. 2015), the majority

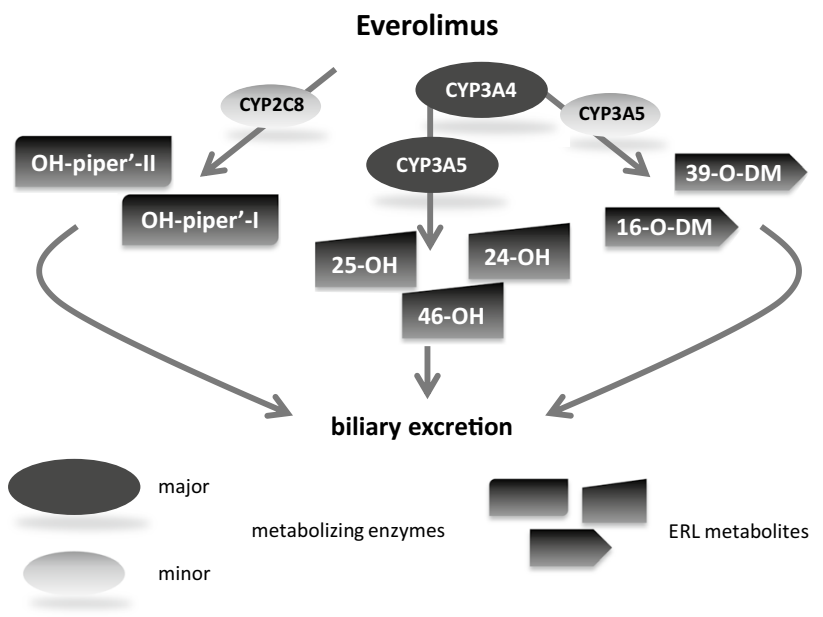

Fig. 1 ERL metabolism in the human liver and gut wall. Enzymes: cytochrome P450 3A5 (CYP3A5), cytochrome P450 3A4 (CYP3A4), cytochrome P450 2C8 (CYP2C8). Metabolites: hydroxypiperidine metabolites I \& II (OH-piper'-I, OH-piper'-II); 24-hydroxy (24-OH), 25-hydroxy (25-OH) and 46-hydroxy (46-OH) ERL; 16-O-desmethyl (16-O-DM) and 39-O-desmethyl (39-O-DM) ERL of the individual variability in ERL dose response remains unexplained (Chapman and Perry 2004).

Metabolomics, the newest of the emerging "omics" fields, studies the entire repertoire of small molecules present in biological samples (Patti et al. 2012). Due to recent technical advances, especially in mass spectrometry (MS), it is currently possible to rapidly measure thousands of metabolites in small sample volumes (e.g. blood plasma) (Fang and Gonzalez 2014). Therefore, metabolic profiles can serve as direct signatures of biochemical activity of an organism, and thus, provide a more direct description of drug response phenotypes than data obtained with other "omics" approaches (Ramautar et al. 2013). Metabolic profiles reflect the combined effect of genetic, environmental, and physiological factors on the individual drug response (Everett 2016). Indeed, it has been successfully shown in the pioneering pharmacometabolomic studies that good and poor therapy responders can be distinguished by screening plasma metabolites (Kaddurah-Daouk et al. 2015; Ji et al. 2011).

We applied a comprehensive pharmacometabolomic profiling technique to address the variability in ERL metabolism and therapy response in cardiac transplant patients. We first performed a targeted quantification of major ERL metabolites in combination with genetic analyses to identify clinical and genetic factors affecting ERL pharmacokinetics and dose requirement. Then, we applied global metabolic profiling to elucidate mechanisms underlying the substantial inter-individual variability in ERL dose response. This study approach may thus provide a new promising strategy to identify factors predictive of individual variability in ERL dose response.

\section{Methods}

\subsection{Patient population}

All patients had received ERL-based maintenance immunosuppressive therapy for at least two months at the time of study recruitment. The targeted ERL $C_{0}$ level was 3-10 ng/ $\mathrm{mL}$ depending on concomitant immunosuppressive therapy. Lithium heparin and EDTA blood samples ( $S$-monovette; Sarstedt, Nümbrecht, Germany) for metabolic profiling were collected in fasting state simultaneously with samples for routine laboratory analyses (i.e., ERL therapeutic drug monitoring). Clinical data, including patient characteristics, ERL daily dose, ERL $C_{0}$ levels, and information about additional immunosuppressive and concomitant drug therapy were retrieved from patient charts. Patients with acute infections, malignancies, or untreated metabolic diseases (i.e., newonset diabetes mellitus, thyroid disease, gout) were excluded from the study. Renal function was assessed by an estimate of the glomerular filtration rate (eGFR) calculated by the 
Chronic Kidney Disease Epidemiology Collaboration equation (Levey et al. 2009).

\subsection{Quantification of ERL and its metabolites in plasma}

EDTA whole blood samples were stored in $1.5 \mathrm{~mL}$ aliquots in CryoPure tubes (Sarstedt, Nümbrecht, Germany) at $-80{ }^{\circ} \mathrm{C}$ until shipment for analysis. Samples were shipped on dry ice to the iC42 Clinical Research and Development Facility at the University of Colorado Denver for quantification of ERL and its major metabolites. Sample preparation and measurement were performed as previously described (Schniedewind et al. 2015; Strom et al. 2007b). Using a validated liquid chromatography coupled tandem mass spectrometry (LC-MS/MS)-based method on an Applied Biosystems/Sciex API4000 triple quadrupole MS (Applied Biosystems, Foster City, CA, USA), ERL and the following metabolites were quantified: 46-hydroxy (46-OH) ERL, 25-OH ERL, 24-OH ERL, 16-O-desmethyl (16-O-DM) ERL, hydroxypiperidine-I (OH-piper'-I) ERL, and 39-ODM ERL. The Analyst software v.1.6.2 (Sciex) was used to control the LC and MS components and process the data. The structures of the ERL metabolites were confirmed as previously described (Strom et al. 2007a; Boernsen et al. 2007).

\subsection{Metabolic profiling}

\subsubsection{Global metabolic profiling}

Lithium heparin blood samples were centrifuged for $15 \mathrm{~min}$ at $2000 \times g$ and $4{ }^{\circ} \mathrm{C}$ and the separated plasma was stored in CapLock Microtubes (Treff AG, Degersheim, Switzerland) at $-140{ }^{\circ} \mathrm{C}$ until final sample preparation and analysis in a randomized order on a high-resolution quadrupole-time-offlight (qTOF-MS; Synapt G2-S HDMS, Waters, Milford, MA, USA) coupled to an ultra-performance liquid chromatography (UPLC) Acquity system (Waters). The samples were introduced by electrospray ionization operating in either negative ion (ESI-) or positive ion (ESI+) mode. The instrument was controlled via MassLynx v.4.1 (Waters). Raw data detection, run alignment, peak picking, and ion deconvolution were performed using Progenesis QI (Nonlinear Dynamics, Newcastle upon Tyne, UK). Additional information on the methods can be found in the Supplementary Material.

\subsubsection{Targeted profiling of glycerophospholipids}

Glycerophospholipids were quantified in heparin plasma samples stored at $-140{ }^{\circ} \mathrm{C}$ by using the flow-injection analysis based MS/MS assay AbsoluteIDQ ${ }^{\circledR}$ p180 (Biocrates Life
Sciences AG, Innsbruck, Austria). Metabolites were quantified using a Xevo TQ-S instrument (Waters). MetIDQ ${ }^{\circledR}$ software (Biocrates Life Sciences AG) was used to manage plate set-up, peak integration as well as concentration calculation. Additional information is provided in the Supplementary Material.

\subsection{Genetic analysis}

Genomic DNA was isolated from EDTA blood samples (4 mL, stored at $-20{ }^{\circ} \mathrm{C}$ ) using QIAamp DNA Blood Midi Kit (Qiagen AG, Basel, Switzerland). The variant CYP3A5*3 was genotyped using a TaqMan ${ }^{\circledR}$ SNP Genotyping Assay (Assay ID C_26201809_30; Thermo Fisher Scientific, Waltham, MA, USA) as previously described (Lesche et al. 2015). Genotype frequencies were tested for deviations from Hardy-Weinberg equilibrium using exact tests implemented in the software Arlequin v.3.5.1.3 (Excoffier and Lischer 2010).

\subsection{Statistical analyses}

ERL and the corresponding metabolite concentrations were used to calculate metabolite/ERL concentration ratios. The dose-adjusted ERL $C_{0}$ levels (i.e., ERL $C_{0}$ level divided by the daily ERL dose per $\mathrm{kg}$ ) were calculated using ERL $C_{0}$ and the daily dose at the day of sampling retrieved from the patient chart. The dose-adjusted ERL $C_{0}$ level and the metabolite/ERL concentration ratios were normalized by logarithmic transformation for further analysis. Normal distribution of continuous clinical data was assessed by Kolmogorov-Smirnov test and data were presented as mean $( \pm \mathrm{SD})$ or as median (min-max) where appropriate.

Associations of demographic and clinical factors (i.e., age, sex, time on ERL therapy, time after transplantation, lipoprotein levels, total cholesterol, triglyceride levels, and eGFR) and the genetic variant $C Y P 3 A 5^{*} 3$ with the doseadjusted ERL $C_{0}$ and the concentration ratios were evaluated using analysis of variance (ANOVA) and Student's t test for group-wise comparisons and linear regression and Pearson's correlation for continuous variables. The influence of potentially interacting co-medication (Furger and Suter 2009; Wessler et al. 2013) on the normalized dose-adjusted ERL $C_{0}$ levels and daily ERL doses was assessed using Student's $\mathrm{t}$ test and Mann-Whitney U test, respectively. Multiple linear regression modelling was used to identify clinical factors independently associated $(P \leq 0.05)$ with the dose-adjusted ERL $C_{0}$ and used as covariates in non-targeted metabolite data analyses. Described statistical analyses were performed using IBM SPSS v.21.0 (IBM Corp., Armonk, NY).

Raw abundance data from non-targeted metabolic profiling were normalized using a global normalization method over all measured compounds as implemented in Progenesis 
QI (Nonlinear Dynamics). Obtained metabolic features were filtered according to peak width, charge, coefficient of variation in quality control (QC) samples, retention time, interfering co-medication, and interferences from the blood collection tubes as described in detail in Supplementary Material. Surveillance of system measurement stability was performed by visually checking for outliers in the QC samples in principal component analysis (PCA) with Pareto scaled data as implemented in SIMCA v.14.0 (MKS Data Analytics Solutions, Umeå, Sweden; Supplementary Fig. 1). For statistical evaluation of the metabolite data, the R software v.3.2 (http://www.r-project.org) was used. The normalized doseadjusted ERL $C_{0}$ level was set as a dependent variable, while metabolite peak data (i.e., normalized abundance) were set as predictor variables. For subsequent exhaustive modelling, a set of 50 best predictive variables was selected by car-scoring (i.e., computing correlation coefficients between the dependent and the Mahalanobis-decorrelated predictor variables) to limit the size of the model space (Kessey et al. 2015; Burnham and Anderson 2002). Modelling was performed using a standard glm function in $\mathrm{R}$ and exhaustive search for the best subsets of variables was performed using the package 'leaps' (Hernandez-Ruedas et al. 2014). The models were subsequently ranked according to Akaike's Information Criterion (AIC) and the best model was chosen. Metabolic features remaining in the final model were revised for peak symmetry, all-over peak abundance and background noise, and peak distribution in all samples (i.e., clear peak in at least three patient samples) with Progenesis QI (Nonlinear Dynamics). Univariate linear regressions as well as linear regressions adjusted for clinical and genetic variables for each metabolic feature were calculated using SPSS. Putative metabolic features were annotated using the Human Metabolome Data Base (HMDB) (Wishart et al. 2013), LIPID MAPS (Fahy et al. 2007), KEGG (http://www.kegg. jp/kegg/), Metlin (Smith et al. 2005), and MetFrag (Wolf et al. 2010), assuming a $\Delta \mathrm{m} / \mathrm{z} \leq 10 \mathrm{ppm}$. Levels of metabolite identification were defined as described in Sumner et al. (2007).

\section{Results}

The study cohort consisted of 41 cardiac transplant patients recruited at the University Hospital Bern. A subset of the patients $(23 / 41 ; 56 \%)$ was also included in a previous study on ERL pharmacogenomics (Lesche et al. 2015). The median age of the patients was 56 years and the majority were males $(29 / 41 ; 71 \%)$ and of Caucasian ethnicity (98\%). On average, patients had been treated with ERLbased maintenance therapy for 4.2 years at the time of study recruitment (Table 1). Four patients $(10 \%)$ were treated with additional low-dose tacrolimus and $37(90 \%)$ patients
Table 1 Characteristics of the study cohort

\begin{tabular}{ll}
\hline Variable & $\mathrm{n}=41$ \\
\hline Time on ERL (years) & $4.2( \pm 2.8)$ \\
Time from transplantation (years) & $6.1(0.3-21.4)$ \\
Age $($ years $)$ & $56.3(20.0-79.0)$ \\
BMI $\left(\mathrm{kg} / \mathrm{m}^{2}\right)$ & $25.0( \pm 4.5)$ \\
eGFR $\left(\mathrm{mL} / \mathrm{min} / 1.73 \mathrm{~m}^{2}\right)$ & $59.8(22.6-148.7)$ \\
LDL $(\mathrm{mmol} / \mathrm{L})$ & $3.0( \pm 1.0)^{\mathrm{a}}$ \\
Total cholesterol $(\mathrm{mmol} / \mathrm{L})$ & $5.4( \pm 1.3)^{\mathrm{b}}$ \\
Triglycerides $(\mathrm{mmol} / \mathrm{L})$ & $1.9(0.8-10.3)^{\mathrm{b}}$ \\
ERL $C_{0}(\mathrm{ng} / \mathrm{mL})$ & $6.4(4.1-13.8)$ \\
ERL daily dose $(\mathrm{mg})$ & $2.5(0.8-7.3)$ \\
ERL daily dose/weight $(\mathrm{mg} / \mathrm{kg})$ & $0.04(0.01-0.10)$ \\
Dose-adjusted ERL $C_{0}(\mathrm{ng} / \mathrm{mL}$ per $\mathrm{mg} / \mathrm{kg} /$ day $)$ & $169.7(52.7-619.2)$ \\
\hline
\end{tabular}

Continuous data are presented as mean $( \pm \mathrm{SD})$ or as median (minmax) where appropriate

$B M I$ body mass index, $C_{0}$ trough level, $E R L$ everolimus, $e G F R$ estimated glomerular filtration rate, $L D L$ low-density lipoprotein

${ }^{\mathrm{a}} \mathrm{n}=35$

${ }^{b} \mathrm{n}=39$

with mycophenolic acid (MPA) derivatives, which was the prodrug mycophenolate mofetil (MMF; Cellcept ${ }^{\circledR}$ ) for 21 patients and the enteric-coated active sodium mycophenolic acid (Na-MPA, Myfortic $\left.{ }^{\circledR}\right)$ for 16 patients. Twentyeight patients $(68 \%)$ received additional immunosuppression with prednisone [median daily dose $4.4(2.5-15.0) \mathrm{mg}$ ]. The co-administration of other drugs potentially interacting with ERL pharmacokinetics is shown in Table 2. Eight patients with insulin-dependent diabetes mellitus received parenteral short- and long-acting insulin analogues. The frequency of the genetic variant $C Y P 3 A 5 * 3$ was $88 \%$ and the observed genotype frequencies showed no deviation from Hardy-Weinberg equilibrium $(P=0.08)$.

\subsection{Clinical and genetic factors associated with ERL pharmacokinetics}

The dose-adjusted ERL trough $\left(C_{0}\right)$ levels showed over tenfold variability in our patient cohort with a median of 169.7 (52.7-619.2) $\mathrm{ng} / \mathrm{mL}$ per $\mathrm{mg} / \mathrm{kg} /$ day. It was used as a quantitative surrogate marker for ERL dose response in subsequent analyses. Four out of the six measured ERL metabolites were observed (Fig. 2). The main metabolites 46-hydroxy (46-OH) ERL and 24-hydroxy (24-OH) ERL accounted together for $71 \%$ of all ERL metabolites in whole blood. Although 46-OH ERL showed predominantly the highest abundance, 24-OH ERL was the main metabolite observed in six patients (Fig. 2).

The co-administration of Na-MPA was associated with lower dose-adjusted ERL $C_{0}$ levels compared to treatment 
Table 2 Medication potentially interacting with ERL pharmacokinetics

\begin{tabular}{|c|c|c|c|}
\hline Therapeutic Class & Drug(s) & Interaction & $\mathrm{n}=41$ \\
\hline Immunosuppression & Tacrolimus & SUB-3A/SUB-PGP & $4(9.8 \%)$ \\
\hline Antibiotics & Trimethoprim & SUB-2C8/INH-2C8 & $33(80.5 \%)$ \\
\hline \multirow[t]{2}{*}{ Anticoagulant } & Phenprocoumon, acenocoumarol & SUB-3A & $8(19.5 \%)$ \\
\hline & Clopidogrel & SUB-PGP & $6(14.6 \%)$ \\
\hline \multirow[t]{2}{*}{$\mathrm{Ca}^{2+}$-channel blocker } & Diltiazem & INH-3A, INH-PGP & $4(9.8 \%)$ \\
\hline & Amlodipine & SUB-3A & $5(12.2 \%)$ \\
\hline \multirow[t]{2}{*}{$\mathrm{H}_{1}$-antagonist } & Es- and omeprazole & SUB-3A/INH-3A & $19(46.3 \%)$ \\
\hline & Pantoprazole & SUB-3A & $9(22.0 \%)$ \\
\hline \multirow[t]{2}{*}{ Lipid-lowering agent } & Ezetimibe & SUB-PGP & $16(39.0 \%)$ \\
\hline & Atorvastatin, simvastatin ${ }^{a}$ & SUB-3A & $6(14.6 \%)$ \\
\hline
\end{tabular}

INH-3A, moderate CYP3A4/5 inhibitor; INH-2C8, moderate CYP2C8 inhibitor; INH-PGP ,inhibitor of P-glycoprotein; SUB-3A, substrate of CYP3A4/5; SUB-2C8, substrate of CYP2C8; SUB-PGP, substrate of P-glycoprotein

${ }^{a}$ All other patients received fluvastatin, pravastatin or rosuvastatin which are not described to interact with CYP3A4/5, CYP2C8 or P-glycoprotein

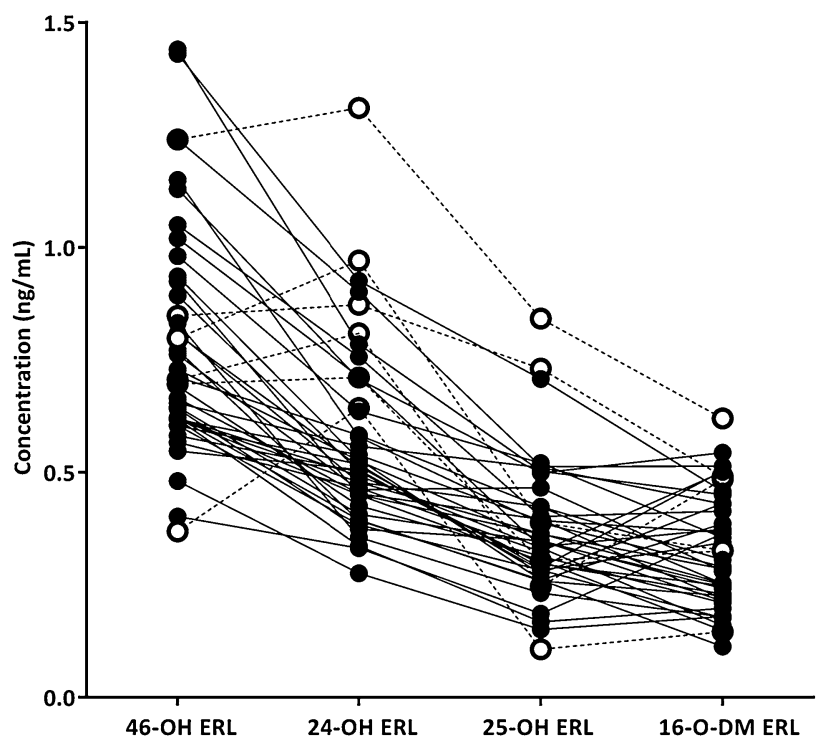

Fig. 2 Individual concentration profiles of ERL metabolites in whole blood samples of 41 cardiac transplant patients. Six patients (dashed line, open circles) showed higher 24-OH ERL concentrations compared to 46-OH ERL, with lines connecting the metabolite data points of one individual. Metabolites: 46-hydroxy everolimus (46-OH ERL), 24-hydroxy everolimus (24-OH ERL), 25-hydroxy everolimus (25-OH ERL), 16-O-desmethyl everolimus (16-O-DM ERL)

with the MMF ( $P=0.013$; Fig. 3a). This difference was translated to a higher daily ERL dose needed by patients treated with Na-MPA to reach their targeted $C_{0}$ level $(P=0.008$; Fig. 3 b). Co-administration of vitamin $\mathrm{K}$ antagonists (i.e., phenprocoumon, or acenocoumarol) was associated with higher dose-adjusted ERL $C_{0}$ levels compared to patients without this treatment, although the effect did not reach statistical significance $(P=0.135)$. CYP3A5 expressors (i.e., patients with $C Y P 3 A 5^{*} 1 / * 3$ and $C Y P 3 A 5^{*} 1 / * 1$ genotypes) showed slightly lower dose-adjusted ERL $C_{0}$ levels $(P=0.074)$ and received slightly higher ERL daily doses $(P=0.059)$ compared to CYP3A5 non-expressors (i.e., CYP $3 A 5^{*} 3 / * 3$ genotype; Fig. 3c, d). Additionally, an association of lower dose-adjusted ERL $C_{0}$ levels, indicative of an increased ERL dose requirement, with elevated low-density lipoprotein $(\mathrm{LDL})$ levels was observed $\left(\mathrm{r}_{\mathrm{PEARSON}}=-0.348\right.$; $P=0.041$ ). None of the factors associated with the doseadjusted ERL $C_{0}$ level showed an effect on ERL metabolite formation or the metabolite/ERL concentration ratios (data not shown). Higher 46-OH/ERL ratios were, however, observed in older patients $\left(\mathrm{r}_{\text {PEARSON }}=0.360 ; P=0.021\right)$.

The $C Y P 3 A 5^{*} 3$ genotype and co-medication with $\mathrm{Na}$ MPA and vitamin $\mathrm{K}$ antagonists were included as covariates in the regression models of non-targeted metabolite data, since these variables were independently associated with the dose-adjusted ERL $C_{0}$ level in a multivariate regression model $\left(R^{2}=0.345 ; P=0.001\right)$. None of the other demographic or clinical factors showed an association with the dose-adjusted ERL $C_{0}$ levels or metabolite/ERL concentration ratios.

\subsection{Global metabolic profiling}

Non-targeted metabolic profiling revealed a total of 1634 and 718 ion features using positive and negative modes of ionization, respectively (Supplementary Table 1). All ion features were subjected to statistical analysis as predictor variables. Because ERL (calculated exact mass $957.5814 \mathrm{Da}$ ) and associated $[\mathrm{M}+\mathrm{H}]^{+},[\mathrm{M}+\mathrm{Na}]^{+}$, and $[\mathrm{M}+\mathrm{K}]^{+}$adducts were not detected using our LC-MS method when injecting analytical standards (data not shown), it was unlikely that related ions were included in the data set.

Including ion features identified in positive ionization mode, the model with the best fit obtained with exhaustive linear regression modelling contained eight ions, from 
Fig. 3 Differences in the dose-adjusted ERL $C_{0}$ levels and ERL dose in patients with co-administration of different MPA derivatives, i.e., mycophenolate mofetil (MMF, $\mathrm{n}=21$ ) and sodium mycophenolic acid (Na-MPA; $\mathrm{n}=16)(\mathbf{a}-\mathbf{b})$ and between CYP3A5 non-expressors $(C Y P 3 A 5 * 3 / * 3)$ and CYP3A5 expressors $\left(C Y P 3 A 5^{*} 1 / * 3\right.$ and CYP $\left.3 A 5^{*} 1 / * 1\right)(\mathbf{c}-\mathbf{d})$. The bottom and top of the box represent the first and third quartiles, and the band inside is the median. Whiskers indicate minimum and maximum
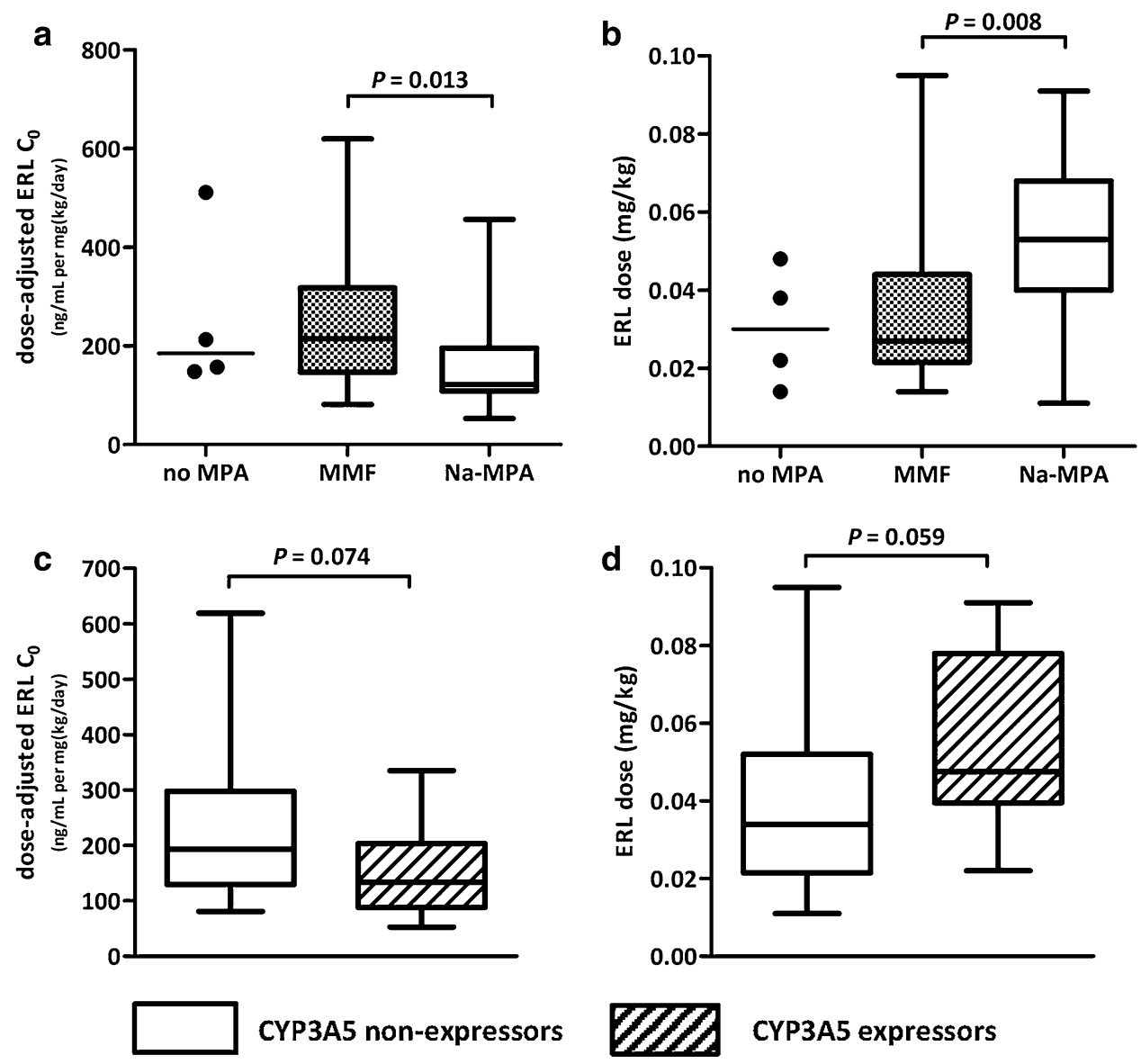

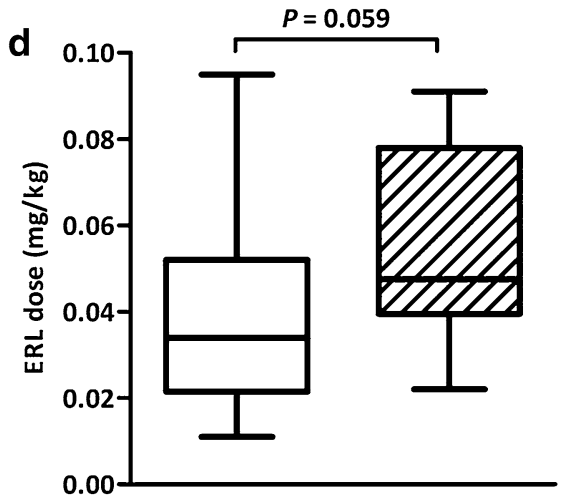

CYP3A5 expressors which six showed an acceptable peak shape and intensity in subsequent visual inspection (Supplementary Material, Table 3). Four ions showed associations with dose-adjusted ERL $C_{0}$ levels independent of clinical and genetic factors. A linear regression model including these four features and the covariates accounted for $71 \%$ of the variability in the dose-adjusted ERL $C_{0}$ levels $\left(R^{2}=0.714 ; P<0.001\right)$. The ion with a peak at $3.54 \mathrm{~min}(136.0744 \mathrm{~m} / \mathrm{z})$ was the main contributor to this variability (partial $R^{2}=0.179$ ), with high normalized peak abundance associated with increased doseadjusted ERL $C_{0}$ levels (Fig. 4a). Although the feature could be identified as a compound with the sum formula $\mathrm{C}_{8} \mathrm{H}_{9} \mathrm{NO}$, a more precise identification was not possible due to missing recording of $\mathrm{MS}^{\mathrm{E}}$ fragmentation data (MSI identification level 3). The other independently associated features could not be annotated by database search taking $\mathrm{MS}^{\mathrm{E}}$ data into account (Supplementary Fig. 3). The ion feature detected at $7.01 \mathrm{~min}(415.1706 \mathrm{~m} / \mathrm{z})$ was identified as the calcium channel blocker diltiazem based on a database match and confirmation by $\mathrm{MS}^{\mathrm{E}}$ fragmentation data (HMDB 14487; MSI identification level 2). In this cohort, four patients were reported to be treated with diltiazem (Table 2), and from these patients, three showed high abundance peaks of this compound associated with increased dose-adjusted ERL $C_{0}$ levels. The compound was not detected in any other patient.

Similarly, for negative ionization mode, the model with the best fit obtained with exhaustive linear regression modelling contained eight ion features, which all showed an acceptable peak shape and intensity (Table 3 ). Three features were independently associated with the dose-adjusted ERL $C_{0}$ level, when the analysis was adjusted for clinical and genetic co-variables. Normalized peak abundance of these three metabolites was positively correlated with the dose-adjusted ERL $C_{0}$ level (Fig. $4 \mathrm{e}-\mathrm{g}$ ). A linear regression model including the three independently associated metabolites and the co-variables explained $70 \%$ of the variability in the dose-adjusted ERL $C_{0}$ levels $\left(R^{2}=0.703 ; P<0.001\right)$. These metabolic features, however, could not be identified based on a database search (MSI identification level 4) using available $\mathrm{MS}^{\mathrm{E}}$ data (Supplementary Fig. 4).

More than $85 \%$ of the inter-individual variability in doseadjusted ERL $C_{0}$ levels could be explained with a multivariate regression model $\left(R^{2}=0.855 ; \mathrm{P}<0.001\right)$ including all independently associated clinical and genetic factors and ion features measured in positive and negative ionization mode. The model also revealed a partial correlation between ion 
Table 3 Metabolic features detected with positive and negative ionization mode associated with dose-adjusted everolimus trough levels

\begin{tabular}{|c|c|c|c|c|c|c|}
\hline \multirow[t]{2}{*}{ Retention time } & \multirow[t]{2}{*}{ Ion } & \multicolumn{2}{|c|}{$\begin{array}{l}\text { Univariate linear } \\
\text { regression }\end{array}$} & \multicolumn{2}{|c|}{$\begin{array}{l}\text { Multi- } \\
\text { variate linear } \\
\text { regression }\end{array}$} & \multirow[t]{2}{*}{ Annotation } \\
\hline & & $\overline{R^{2}}$ & $P$ & $\overline{R^{2}}$ & $P$ & \\
\hline \multicolumn{7}{|c|}{ Positive ionization mode } \\
\hline $3.54 \mathrm{~min}$ & $136.0744 \mathrm{~m} / \mathrm{z}$ & 0.270 & $<0.001$ & 0.467 & 0.005 & Chemical formula: $\mathrm{C}_{8} \mathrm{H}_{9} \mathrm{NO}$ \\
\hline $3.79 \mathrm{~min}$ & $316.2110 \mathrm{~m} / \mathrm{z}$ & 0.190 & 0.004 & 0.438 & 0.014 & None \\
\hline $4.23 \mathrm{~min}$ & $247.0878 \mathrm{n}^{\mathrm{a}}$ & 0.105 & 0.039 & 0.393 & 0.069 & None \\
\hline $6.48 \mathrm{~min}$ & $599.2454 \mathrm{~m} / \mathrm{z}$ & 0.102 & 0.042 & 0.405 & 0.045 & None \\
\hline $6.63 \mathrm{~min}$ & $232.1296 \mathrm{n}^{\mathrm{a}}$ & 0.124 & 0.024 & 0.421 & 0.026 & None \\
\hline $7.01 \mathrm{~min}$ & $415.1706 \mathrm{~m} / \mathrm{z}$ & 0.041 & 0.205 & 0.380 & 0.112 & Diltiazem \\
\hline \multicolumn{7}{|c|}{ Negative ionization mode } \\
\hline $5.65 \mathrm{~min}$ & $229.0162 \mathrm{~m} / \mathrm{z}$ & 0.058 & 0.130 & 0.349 & 0.375 & None \\
\hline $6.94 \mathrm{~min}$ & $461.0052 \mathrm{n}^{\mathrm{a}}$ & 0.082 & 0.070 & 0.385 & 0.094 & None \\
\hline $7.82 \mathrm{~min}$ & $525.2686 \mathrm{~m} / \mathrm{z}$ & 0.040 & 0.208 & 0.338 & 0.084 & None \\
\hline $7.90 \mathrm{~min}$ & $391.1824 \mathrm{~m} / \mathrm{z}$ & 0.114 & $\mathbf{0 . 0 3 1}$ & 0.409 & 0.039 & None \\
\hline $8.31 \mathrm{~min}$ & $427.2143 \mathrm{~m} / \mathrm{z}$ & 0.238 & 0.001 & 0.405 & 0.046 & None \\
\hline $9.23 \mathrm{~min}$ & $427.2145 \mathrm{~m} / \mathrm{z}$ & 0.142 & 0.015 & 0.378 & 0.120 & None \\
\hline $9.27 \mathrm{~min}$ & $550.2438 \mathrm{~m} / \mathrm{z}$ & 0.178 & 0.006 & 0.460 & 0.006 & None \\
\hline $10.91 \mathrm{~min}$ & $480.3091 \mathrm{~m} / \mathrm{z}$ & 0.197 & 0.004 & 0.400 & 0.054 & Lysophosphatidylcholine 16:0/0:0 \\
\hline
\end{tabular}

${ }^{a}$ Calculated neutral mass based on detected adduct ions at the same retention time

${ }^{\text {b }}$ Adjusted for $C Y P 3 A 5^{*} 3$ genotype and therapy with Na-MPA and vitamin K antagonists

${ }^{\mathrm{c}} \mathrm{C}_{24} \mathrm{H}_{50} \mathrm{NO}_{7} \mathrm{P}$, loss of a methyl group in negative mode

Bold font indicates $P<0.05$ features indicating that they may belong to the same metabolic pathway (Supplementary Fig. 2).

Interestingly, a metabolite detected at $10.91 \mathrm{~min}$ $(480.3091 \mathrm{~m} / \mathrm{z})$, that was included in the original best-fit model, was tentatively identified as a lysophosphatidylcholine (16:0/0:0) [lysoPC (16:0/0:0); LMGP01050018] that showed a strong negative correlation with the dose-adjusted ERL $C_{0}$ level (Table 3 and Fig. 4i). The identification of lysoPC (16:0/0:0) was confirmed by comparing its retention time and $\mathrm{MS}^{\mathrm{E}}$ fragmentation data to those of an analytical standard using the same non-targeted method as above (MSI identification level 2, Supplementary Fig. 4i). Both showed a peak at $255.2331 \mathrm{~m} / \mathrm{z}$ representing the $[\mathrm{M}-\mathrm{H}]^{-}$ion of palmitic acid, the fatty acid residue present in lysoPC (16:0/0:0).

\subsection{Targeted profiling of glycerophospholipids}

To further explore the association of the identified lysoPC (16:0/0:0) as well as other lysoPC compounds with ERL dose requirement, glycerophospholipid profiling was performed using the Absolute $I D Q^{\circledR}$ p180 kit. In agreement with our previous results, the plasma concentration of lysoPC (16:0/0:0) showed a strong correlation with the normalized peak abundance of lysoPC (16:0/0:0) determined with the non-targeted approach $\left(\mathrm{r}_{\mathrm{PEARSON}}=0.886 ; P<0.001\right)$. It also showed a similar negative correlation with the normalized dose-adjusted ERL $C_{0}$ level (Fig. $4 \mathrm{~h}$ ). Additionally, two other lysoPC compounds, lysoPC (17:0/0:0) and lysoPC (18:0/0:0), showed a similar association with the doseadjusted ERL $C_{0}$ level $\left(\mathrm{r}_{\text {PEARSON }}=-0.536 ; P<0.001\right.$ and $\mathrm{r}_{\text {PEARSON }}=-0.455 ; P=0.002$, respectively).

\section{Discussion}

Although ERL is increasingly used in immunosuppressive therapy after cardiac transplantation, patients' substantial variability in dose response remains a major hurdle for achieving successful therapy. The biological mechanisms underlying this variability are poorly understood, and biomarkers predictive of individual ERL dose requirement for optimizing the therapy for each patient are still lacking. In this study, we combined quantification of drug metabolites and metabolic profiling to evaluate factors affecting ERL metabolism and to identify new metabolic biomarkers associated with individual ERL dose response. By quantifying ERL and its metabolites in blood samples of 41 cardiac transplant patients, we observed the influence of co-medication and the genetic variant $C Y P 3 A 5^{*} 3$ on the daily ERL dose requirement. By using global plasma metabolic profiling and a targeted analysis of glycerophospholipids, we were 

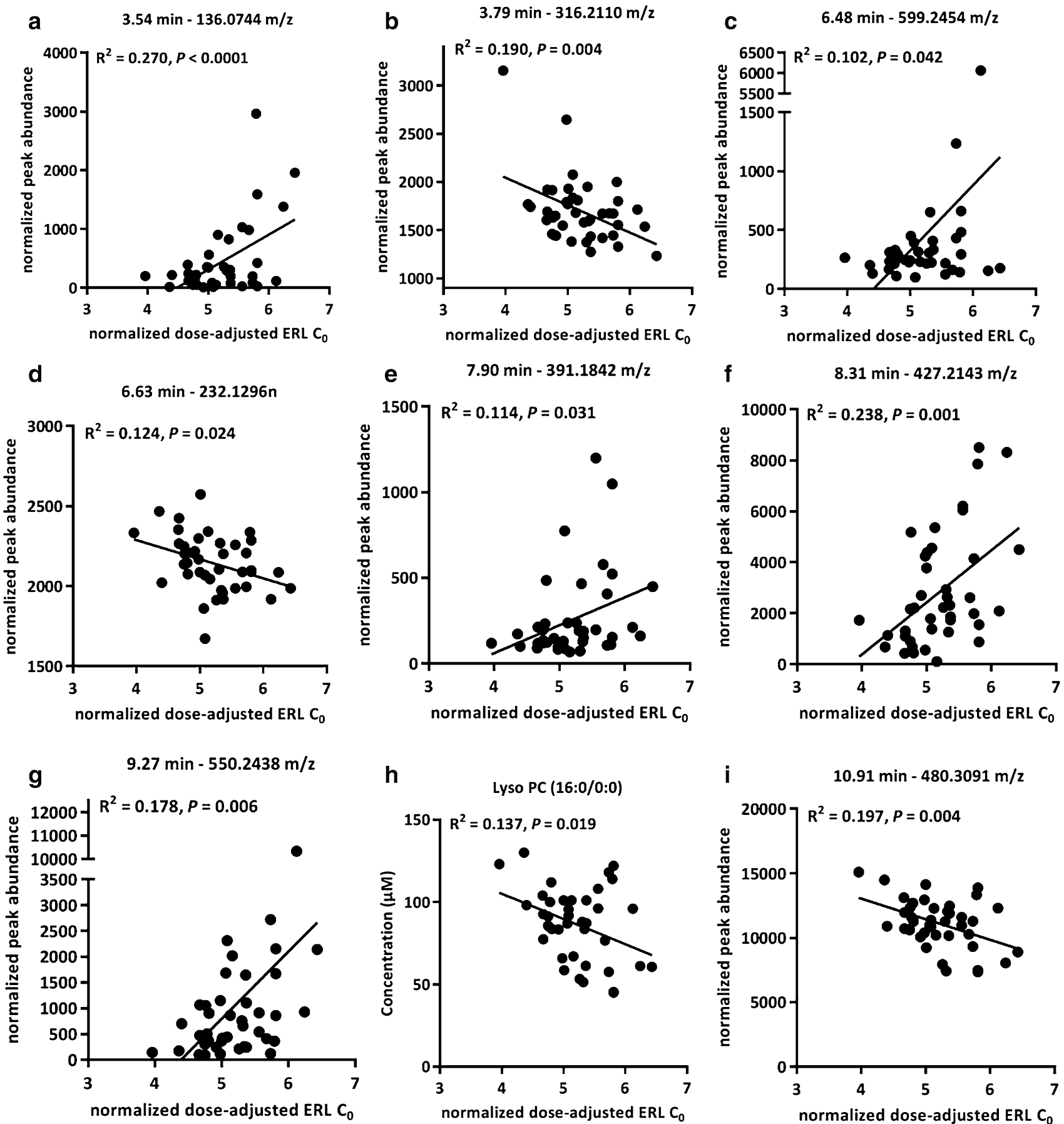

h

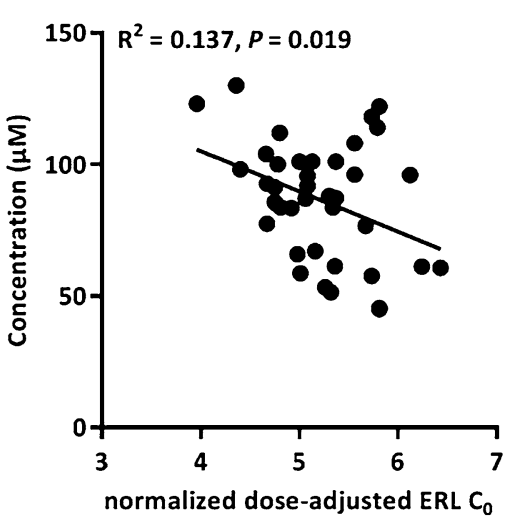

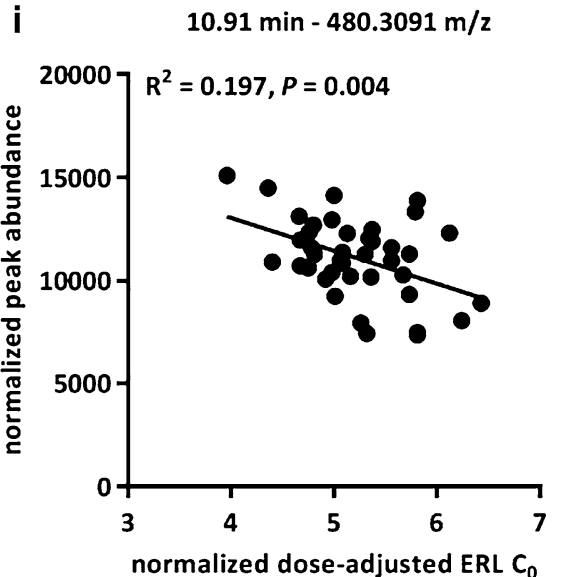

Fig. 4 Univariate regression of metabolic features independently associated with the dose-adjusted ERL $C_{0}$ levels. Metabolic features were identified with positive (a-d) and negative $(\mathbf{e}-\mathbf{g})$ ionization mode in non-targeted metabolic profiling. Lysophosphatidylcholine
(16:0/0:0) identified with the targeted AbsoluteIDQ p180 kit (h) and the non-targeted method in negative ionization mode at $10.91 \mathrm{~min}$ and $480.3091 \mathrm{~m} / \mathrm{z}(\mathbf{i})$ able to identify lysoPCs as metabolites associated with ERL dose response.

The main metabolic pathway of ERL is mediated by CYP3A4 and CYP3A5 producing predominantly hydroxylated ERL metabolites (Fig. 1). The 46-OH ERL was the major metabolite observed in the majority of our cardiac transplant patients. Interestingly, six patients (15\%) showed an increased formation of the 24-OH ERL metabolite. The pharmacological and toxicological properties of ERL metabolites are currently unknown but they might be of clinical 
relevance, especially regarding adverse drug effects. Strom et al. hypothesized that $C Y P 3 A 5$ genotype may contribute to differences in the formation of ERL metabolites (Strom et al. 2007b). Although we showed that the CYP3A5*3 variant might have a minor effect on ERL dose requirement, it was not associated with differences in ERL metabolite patterns in our patient cohort. These differences were also not explained by the patients' age, although an association of age with 46-OH ERL/ERL concentration ratio was observed, most likely reflecting lower ERL $C_{0}$ levels in elderly patients $\left(\mathrm{r}_{\text {PEARSON }}=-0.317 ; P=0.043\right)$.

In this study, we report for the first time differences in the ERL dose requirement between cardiac transplant patients receiving the two different MPA derivatives, Na-MPA and MMF. Patients receiving Na-MPA therapy required significantly higher ERL doses to achieve their targeted blood levels compared to patients receiving MMF or no MPA derivative at all. ERL and MPA derivatives are frequently used in combination for immunosuppressive therapy after heart transplantation but interaction between the drugs has not been described earlier (Schweiger et al. 2012; Hollis et al. 2015). In our patient cohort, Na-MPA was usually prescribed for patients suffering from severe gastrointestinal adverse effects under previous MMF therapy (Jasiak and Park 2016), while ERL therapy remained unchanged under this condition. Although co-administration of Na-MPA therapy changed ERL dose requirement, it had no influence on the ERL metabolite patterns, which might suggest an interaction with ERL absorption. Further investigations are thus needed regarding the pharmacological background of this interaction and its clinical relevance. Similarly, the observed association between anticoagulation therapy with phenprocoumon or acenocoumarol and decreased ERL dose requirement needs further exploration. These drugs are reported to be substrates of CYP3A4 and CYP3A5 enzymes and exhibit extensive plasma protein binding (Ufer et al. 2004; Otagiri et al. 1980). Interaction with ERL might be caused by competitive metabolism or protein binding in plasma but has not been described earlier.

Furthermore, we were able to detect diltiazem with the non-targeted metabolic profiling in the plasma of three out of four patients recorded to receive this drug. The ERL dose requirement of these patients corresponded with the diltiazem abundance, i.e., a lower dose requirement was associated with higher diltiazem levels. Nevertheless, the dose-adjusted ERL $C_{0}$ levels in these patients were not significantly different from patients without reported diltiazem therapy in a group-wise comparison. In contrast to MPA derivatives and anticoagulants, diltiazem is known to interact with ERL pharmacokinetics by moderate inhibition of CYP3A4- and CYP3A5-mediated metabolism and has been reported to lead to significantly increased dose-adjusted ERL $C_{0}$ levels in lung transplant patients
(Schoeppler et al. 2014). Nevertheless, our study was limited to detect even large interaction effects in pairwise comparisons due to the small sample size and only four patients with diltiazem co-medication, thus diltiazem was only found to contribute to the regression model of metabolic features obtained with positive ionization mode in global metabolic profiling.

The putative identification of the lysoPC (16:0/0:0) by our global metabolic profiling was supported by confirmation with an analytical standard and observation of a similar negative correlation of lysoPC (16:0/0:0) plasma concentration with dose-adjusted ERL $C_{0}$ level using targeted profiling of glycerophospholipids. Two additional lysoPCs, namely lysoPC (17:0/0:0) and lysoPC (18:0/0:0), were identified to be similarly associated with ERL dose requirement through targeted glycerophospholipid analysis. While lysoPC (17:0/0:0) is usually observed only in low levels in human tissue, lysoPC (16:0/0:0) and lysoPC (18:0/0:0) are the most abundant lysoPCs in human plasma and have been reported to differ between lipoproteins (Serna et al. 2015).

The negative correlation observed between lysoPC concentrations and the dose-adjusted ERL $C_{0}$ level indicates an association of lower plasma lysoPC levels with a reduced ERL dose requirement. Circulating lysoPCs are generated by lecithin-cholesterol acyltransferase, which is secreted from the liver, and transfers fatty acids from phosphatidylcholines (PC) to cholesterol. Alternatively, lysoPCs can be produced by lipoprotein-associated phospholipase $\mathrm{A}_{2}\left(\mathrm{Lp}-\mathrm{PLA}_{2}\right)$ by hydrolysis of surface PC of lipoproteins (Schmitz and Ruebsaamen 2010; Matsumoto et al. 2007; Tellis and Tselepis 2009). Lp-PLA 2 activity is associated with LDL levels and has been proposed to be a biomarker for atherosclerotic processes and vascular inflammation, such as CAV, in combination with high levels of lysoPCs (Lavi et al. 2007; Schmitz and Ruebsaamen 2010). We observed that patients requiring lower ERL doses to achieve targeted ERL $C_{0}$ levels also showed lower lysoPC levels independent from lipid-lowering therapy. In contrast, an increased ERL dose requirement was associated with higher lysoPC levels potentially reflecting inflammatory processes triggered by inadequate immunosuppression. Thus, patients with a poor response to ERL might be at a higher risk of developing long-term CAV. These patients might not equally benefit from the generally positive effects of ERL on vascular remodeling after cardiac transplantation (Eisen et al. 2003; Hiemann et al. 2011). Rosing et al. (2013) described increased Lp-PLA 2 activity as a biomarker for oxidative stress potentially involved in the development of CAV in heart transplant recipients on ERLbased therapy. However, it remains to be clarified whether patients with poor ERL dose response equally benefit from anti-proliferative ERL effects compared to good responders. 


\subsection{Concluding remarks}

For the first time in cardiac transplant recipients, different ERL metabolite patterns were described. These patterns were not influenced by $C Y P 3 A 5^{*} 3$ genotype or comedication. The combined quantification of ERL and its metabolites and global metabolic profiling allowed us to detect potentially new drug-drug interactions and novel metabolites associated with ERL dose requirement. Especially the role of lysoPCs as biomarkers for CAV-related inflammatory processes, possibly triggered by inadequate immunosuppression, requires further investigation in order to understand their role in altered ERL dose requirement.

Acknowledgements We thank Barbara Rindlisbacher and Roland Geyer form the Clinical Metabolomics Facility at the Centre of Laboratory Medicine (Bern University Hospital) and Bjoern Schniedewind from the iC42 Clinical Research and Development Facility in Denver for technical support, as well as the personnel of the heart failure outpatient clinic at the Bern University Hospital for assistance in patient recruitment.

Funding The study was funded by grants from the Foundation for Pathobiochemistry and Molecular Diagnostics of the German Society of Clinical Chemistry and Laboratory Medicine to J.S., from the United States National Institutes of Health (NICHD R01 HD070511) to U.C., and from the Katharina Huber-Steiner foundation to P.M.

\section{Compliance with ethical standards}

Conflict of interest All authors declare that they have no conflict of interest directly or indirectly related to the research presented in this manuscript.

\section{References}

Andreassen, A. K., Andersson, B., Gustafsson, F., Eiskjaer, H., Radegran, G., Gude, E., et al. (2014). Everolimus initiation and early calcineurin inhibitor withdrawal in heart transplant recipients: a randomized trial. American Journal of Transplant, 14(8), 1828-1838. https://doi.org/10.1111/ajt.12809.

Azimzadeh, A. M., Lees, J. R., Ding, Y., \& Bromberg, J. S. (2011). Immunobiology of transplantation: impact on targets for large and small molecules. Clinical Pharmacology \& Therapeutics, 90(2), 229-242. https://doi.org/10.1038/clpt.2011.106.

Boernsen, K. O., Egge-Jacobsen, W., Inverardi, B., Strom, T., Streit, F., Schiebel, H. M., et al. (2007). Assessment and validation of the MS/MS fragmentation patterns of the macrolide immunosuppressant everolimus. Journal of Mass Spectrometry, 42(6), 793-802. https://doi.org/10.1002/jms.1215.

Burnham, K. P., \& Anderson, D. R. (2002). Model selection and multimodel inference (1st edn.). New York: Springer.

Chapman, T. M., \& Perry, C. M. (2004). Everolimus. Drugs, 64(8), 861-872 (discussion 873-864).

Eisen, H. J., Tuzcu, E. M., Dorent, R., Kobashigawa, J., Mancini, D., Valantine-von Kaeppler, H. A., et al. (2003). Everolimus for the prevention of allograft rejection and vasculopathy in cardiac-transplant recipients. New England Journal of Medicine, 349(9), 847-858. https://doi.org/10.1056/NEJMoa022171.

Everett, J. R. (2016). From metabonomics to pharmacometabonomics: The role of metabolic profiling in personalized medicine. Frontiers in Pharmacology. https://doi.org/10.3389/fphar.2016.00297.

Excoffier, L., \& Lischer, H. E. (2010). Arlequin suite ver 3.5: A new series of programs to perform population genetics analyses under Linux and Windows. Molecular Ecology Resources, 10(3), 564567. https://doi.org/10.1111/j.1755-0998.2010.02847.x.

Fahy, E., Sud, M., Cotter, D., \& Subramaniam, S. (2007). LIPID MAPS online tools for lipid research. Nucleic Acids Research, 35, W606W612. https://doi.org/10.1093/nar/gkm324.

Fang, Z. Z., \& Gonzalez, F. J. (2014). LC-MS-based metabolomics: An update. Archives of Toxicology, 88(8), 1491-1502. https://doi. org/10.1007/s00204-014-1234-6.

Furger, P., \& Suter, T. M. (2009). SURFmed: Guidelines internal medicine (2nd ed.). Neuhausen: Editions D\&F GmbH.

Hernandez-Ruedas, M. A., Arroyo-Rodriguez, V., Meave, J. A., Martinez-Ramos, M., Ibarra-Manriquez, G., Martinez, E., et al. (2014). Conserving tropical tree diversity and forest structure: the value of small rainforest patches in moderately-managed landscapes. PLOS ONE, 9(6), e98931, https://doi.org/10.1371/journal. pone.0098931.

Hiemann, N. E., Wellnhofer, E., Lehmkuhl, H. B., Knosalla, C., Hetzer, R., \& Meyer, R. (2011). Everolimus prevents endomyocardial remodeling after heart transplantation. Transplantation, 92(10), 1165-1172. https://doi.org/10.1097/TP.0b013e3182332886.

Hollis, I. B., Reed, B. N., \& Moranville, M. P. (2015). Medication management of cardiac allograft vasculopathy after heart transplantation. Pharmacotherapy, 35(5), 489-501. doi:https://doi. org/10.1002/phar.1580.

Jasiak, N. M., \& Park, J. M. (2016). Immunosuppression in solidorgan transplantation: Essentials and practical tips. Critical Care Nursing Quarterly, 39(3), 227-240. https://doi.org/10.1097/ CNQ.0000000000000117.

Ji, Y., Hebbring, S., Zhu, H., Jenkins, G. D., Biernacka, J., Snyder, K., et al. (2011). Glycine and a glycine dehydrogenase (GLDC) SNP as citalopram/escitalopram response biomarkers in depression: Pharmacometabolomics-informed pharmacogenomics. Clinical Pharmacology \& Therapeutics, 89(1), 97-104. https://doi. org/10.1038/clpt.2010.250.

Kaddurah-Daouk, R., \& Weinshilboum, R. \& PharmacometabolomicsResearch-Network (2015). Metabolomic signatures for drug response phenotypes: Pharmacometabolomics enables precision medicine. Clinical Pharmacology \& Therapeutics, 98(1), 71-75, https://doi.org/10.1002/cpt.134.

Kessey, A., Lewin, A., \& Strimmer, K. (2015). Optimal whitening and decorrelation. arXiv: $1512.00809 \mathrm{v} 1$

Kirchner, G. I., Meier-Wiedenbach, I., \& Manns, M. P. (2004). Clinical pharmacokinetics of everolimus. Clinical Pharmacokinetics, 43(2), 83-95. https://doi. org/10.2165/00003088-200443020-00002.

Kovarik, J. M., Eisen, H., Dorent, R., Mancini, D., Vigano, M., Rouilly, M., et al. (2003). Everolimus in de novo cardiac transplantation: Pharmacokinetics, therapeutic range, and influence on cyclosporine exposure. The Journal of Heart and Lung Transplantation, 22(10), 1117-1125.

Lavi, S., McConnell, J. P., Rihal, C. S., Prasad, A., Mathew, V., Lerman, L. O., et al. (2007). Local production of lipoprotein-associated phospholipase A2 and lysophosphatidylcholine in the coronary circulation: association with early coronary atherosclerosis and endothelial dysfunction in humans. Circulation, 115(21), 27152721. https://doi.org/10.1161/CIRCULATIONAHA.106.671420.

Lemaitre, F., Bezian, E., Goldwirt, L., Fernandez, C., Farinotti, R., Varnous, S., et al. (2012). Population pharmacokinetics of everolimus in cardiac recipients: comedications, $\mathrm{ABCB} 1$, and CYP3A5 
polymorphisms. Therapeutic Drug Monitoring, 34(6), 686-694. https://doi.org/10.1097/FTD.0b013e318273c899.

Lesche, D., Sigurdardottir, V., Setoud, R., Englberger, L., Fiedler, G. M., Largiader, C. R., et al. (2015). Influence of CYP3A5 genetic variation on everolimus maintenance dosing after cardiac transplantation. Cliniacl Transplantation, 29(12), 1213-1220. https:// doi.org/10.1111/ctr.12653.

Levey, A. S., Stevens, L. A., Schmid, C. H., Zhang, Y. L., Castro, A. F., Feldman, H. I., et al. (2009). A new equation to estimate glomerular filtration rate. Annals of Internal Medicine, 150(9), 604-612.

Matsumoto, T., Kobayashi, T., \& Kamata, K. (2007). Role of lysophosphatidylcholine (LPC) in atherosclerosis. Current Medicinal Chemistry, 14(30), 3209-3220.

Moes, D. J., Swen, J. J., den Hartigh, J., van der Straaten, T., van der Heide, J. J., Sanders, J. S., et al. (2014). Effect of $C Y P 3 A 4^{*} 22$, $C Y P 3 A 5^{*} 3$, and $C Y P 3 A$ combined genotypes on cyclosporine, everolimus, and tacrolimus pharmacokinetics in renal transplantation. CPT Pharmacometrics \& Systems Pharmacology, 3, e100. https://doi.org/10.1038/psp.2013.78.

Otagiri, M., Fleitman, J. S., \& Perrin, J. H. (1980). Investigations into the binding of phenprocoumon to albumin using fluorescence spectroscopy. Journal of Pharmacy and Pharmacology, 32(7), 478-482.

Patti, G. J., Yanes, O., \& Siuzdak, G. (2012). Innovation: Metabolomics: The apogee of the omics trilogy. Nature Reviews Molecular Cell Biology, 13(4), 263-269. https://doi.org/10.1038/ nrm3314.

Raichlin, E., \& Kushwaha, S. S. (2008). Proliferation signal inhibitors and cardiac allograft vasculopathy. Current Opinion in Organ Transplantation, 13(5), 543-550. https://doi.org/10.1097/ MOT.0b013e32830fdf70.

Ramautar, R., Berger, R., van der Greef, J., \& Hankemeier, T. (2013). Human metabolomics: Strategies to understand biology. Current Opinion in Chemical Biology, 17(5), 841-846. https://doi. org/10.1016/j.cbpa.2013.06.015.

Rosing, K., Fobker, M., Kannenberg, F., Gunia, S., Dell'Aquila, A. M., Kwiecien, R., et al. (2013). Everolimus therapy is associated with reduced lipoprotein-associated phospholipase A2 (Lp$\mathrm{Pla} 2)$ activity and oxidative stress in heart transplant recipients. Atherosclerosis, 230(1), 164-170. https://doi.org/10.1016/j. atherosclerosis.2013.07.007.

Schmitz, G., \& Ruebsaamen, K. (2010). Metabolism and atherogenic disease association of lysophosphatidylcholine. Atherosclerosis, 208(1), 10-18. https://doi.org/10.1016/j. atherosclerosis.2009.05.029.

Schniedewind, B., Niederlechner, S., Galinkin, J. L., Johnson-Davis, K. L., Christians, U., \& Meyer, E. J. (2015). Long-term crossvalidation of everolimus therapeutic drug monitoring assays: the Zortracker study. Therapeutic Drug Monitoring, 37(3), 296-303. https://doi.org/10.1097/FTD.0000000000000191.

Schoeppler, K. E., Aquilante, C. L., Kiser, T. H., Fish, D. N., \& Zamora, M. R. (2014). The impact of genetic polymorphisms, diltiazem, and demographic variables on everolimus trough concentrations in lung transplant recipients. Clinical Transplantation, 28(5), 590-597. https://doi.org/10.1111/ctr.12350.

Schweiger, M., Stiegler, P., Puntschart, A., Sereinigg, M., Prenner, G., Wasler, A., et al. (2012). Everolimus in different combinations as maintenance immunosuppressive therapy in heart transplant recipients. Experimental and Clinical Transplantation, 10(3), 273-277.

Serna, J., Garcia-Seisdedos, D., Alcazar, A., Lasuncion, M. A., Busto, R., \& Pastor, O. (2015). Quantitative lipidomic analysis of plasma and plasma lipoproteins using MALDI-TOF mass spectrometry. Chemistry and Physics of Lipids, 189, 7-18. https://doi. org/10.1016/j.chemphyslip.2015.05.005.

Smith, C. A., O'Maille, G., Want, E. J., Qin, C., Trauger, S. A., Brandon, T. R., et al. (2005). METLIN: A metabolite mass spectral database. Therapeutic Drug Monitoring, 27(6), 747-751.

Strom, T., Haschke, M., Boyd, J., Roberts, M., Arabshahi, L., Marbach, P., et al. (2007a). Crossreactivity of isolated everolimus metabolites with the Innofluor Certican immunoassay for therapeutic drug monitoring of everolimus. Therapeutic Drug Monitoring, 29(6), 743-749. https://doi.org/10.1097/FTD.0b013e31815b3cbf.

Strom, T., Haschke, M., Zhang, Y. L., Bendrick-Peart, J., Boyd, J., Roberts, M., et al. (2007b). Identification of everolimus metabolite patterns in trough blood samples of kidney transplant patients. Therapeutic Drug Monitoring, 29(5), 592-599. https://doi. org/10.1097/FTD.0b013e3181570830.

Sumner, L. W., Amberg, A., Barrett, D., Beale, M. H., Beger, R., Daykin, C. A., et al. (2007). Proposed minimum reporting standards for chemical analysis. Metabolomics, 3(3), 211-221. https:// doi.org/10.1007/s11306-007-0082-2.

Tellis, C. C., \& Tselepis, A. D. (2009). The role of lipoprotein-associated phospholipase A2 in atherosclerosis may depend on its lipoprotein carrier in plasma. Biochim Biophys Acta, 1791(5), 327-338. https://doi.org/10.1016/j.bbalip.2009.02.015.

Ufer, M., Svensson, J. O., Krausz, K. W., Gelboin, H. V., Rane, A., \& Tybring, G. (2004). Identification of cytochromes P450 2C9 and $3 \mathrm{~A} 4$ as the major catalysts of phenprocoumon hydroxylation in vitro. European Journal of Clinical Pharmacology, 60(3), 173-182. https://doi.org/10.1007/s00228-004-0740-5.

Wessler, J. D., Grip, L. T., Mendell, J., \& Giugliano, R. P. (2013). The P-glycoprotein transport system and cardiovascular drugs. Journal of the American College of Cardiology, 61(25), 2495-2502. https://doi.org/10.1016/j.jacc.2013.02.058.

Wishart, D. S., Jewison, T., Guo, A. C., Wilson, M., Knox, C., Liu, Y., et al. (2013). HMDB 3.0-The human metabolome database in 2013. Nucleic Acids Research, 41, D801-D807. https://doi. org/10.1093/nar/gks1065.

Wolf, S., Schmidt, S., Muller-Hannemann, M., \& Neumann, S. (2010). In silico fragmentation for computer assisted identification of metabolite mass spectra. BMC Bioinformatics, 11, 148. https:// doi.org/10.1186/1471-2105-11-148. 